\title{
Density-PDFs and Lagrangian Statistics of highly compressible Turbulence
}

\author{
Christoph Beetz, Christian Schwarz, Jürgen Dreher, and Rainer Grauer \\ Theoretische Physik I, Ruhr-Universität Bochum, Germany
}

(Dated: October 31, 2018)

\begin{abstract}
We report on probability-density-functions (PDF) of the mass density in numerical simulations of highly compressible hydrodynamic flows and the corresponding structure formation of Lagrangian particles advected by the flows. Numerical simulations were performed with $512^{3}$ collocation points and 2 million tracer particles integrated over several dynamical times. We propose a connection between the PDF of the Lagrangian tracer particles and the predicted log-normal distribution of the density fluctuations in isothermal systems.

PACS numbers: 47.11.Df, 47.27.E-, 47.40.Ki, 98.38.Am
\end{abstract}

Introduction: Compressible fluid and plasma turbulence is believed to be of fundamental importance for understanding relevant processes in astrophysics. A typical example is the effect of turbulence on the star formation rate in dense molecular clouds in the interstellar medium [1]. The effect of turbulence is manifested in the highly intermittent nature of density fluctuations in supersonic flows which naturally has an enormous impact on the local conditions triggering star formation.

Traditionally, turbulence is studied in the Eulerian description, where the temporal evolution of the fluid quantities is followed at fixed spatial locations. However, in the last 10 years the Lagrangian description, which describes the motion of fluid particles, has undergone a rapid development. This is mainly due to advanced experimental techniques introduced in Ris $\varnothing$ 2], Cornell $[3,4,45]$ and Lyon [6] for studying the statistics of passive tracer particles in incompressible turbulent fluids. Both, experimental and numerical [7, 8] studies highlight the importance of singular structures like vortex tubes and sheets for the intermittent statistics of incompressible flows. Lagrangian statistics is not only interesting for obtaining a deeper understanding of the influence of typical coherent or nearly-singular structures in the flow but also of fundamental importance for understanding mixing, clustering and diffusion properties of turbulent astrophysical fluid and plasma flows.

In this Letter, we will use the Lagrangian description of passive tracer particles in highly compressible flows. Due to the compressibility, the situation is very different from the incompressible case. Clustering of passive tracer particles will reflect the strong density fluctuations. This effect does not appear in the incompressible case. However, the incompressible case will serve as a guideline to obtain a mapping between the PDF of density fluctuations and the PDF of the particle distribution.

The goal of the paper is threefold: The main result is an analytical expression for the spatial distribution of the Lagrangian tracers. This result will be used to confirm and quantify the relation between the standard deviation of the Eulerian density fluctuations and the mean Mach number. And last, the analytical description helps to define a precise structuring time for the clustering of the Lagrangian particles which turns out to be significantly shorter than the dynamical time.

Numerical Methods: To follow this approach in the examination of compressible fluid turbulence we solved the isothermal Euler equations for density $\rho$ and momentum density $\mathbf{u}=\rho \mathbf{v}$

$$
\begin{aligned}
\partial_{t} \rho+\nabla \cdot \mathbf{u} & =0 \\
\partial_{t} \mathbf{u}+\nabla \cdot\left(\frac{\mathbf{u u}}{\rho}\right) & =-\nabla P+\rho \mathbf{k}
\end{aligned}
$$

with $P \sim \rho$ using direct numerical simulations in real space, and realized Lagrangian measurements with tracer particles, which are advected by the simulated flow. We performed three runs of stationary flows ( $a, b$ and $c$ ) with mean r.m.s. Mach numbers $\tilde{M}$ of $0.4,1.4$ and 4.6, respectively.

Equations (11) were solved in the racoon environment for hyperbolic differential equations $[9]$. It utilizes a third order semi-discrete central scheme [10]. Integration is done by a third order strongly stable Runge-Kutta scheme [11]. A 3-dimensional cube of length $L=2 \pi$ was discretized in physical space with $\mathcal{N}^{3}=512^{3}$ collocation points and periodic boundary conditions. The simulation was started with an Orszag-Tang like initial condition and a homogeneous density. The simulations were run for several dynamical times $t_{d y n}=L / 2 M$ to let the system evolve into a turbulent state. The mean energy input was realized by a temporal delta correlated stirring force with zero mean momentum. Forcing was applied in a shell $k \in[1,2]$ in Fourier space, such that it acts only on scales comparable to the entire box. The amplitudes of the Fourier modes were adjusted in order to achieve stationarity at various mean Mach numbers. Then the mean energy input was kept constant for each run.

In this evolved turbulent system we inserted $N=2000000$ particles. They were randomly distributed over the computational domain and then advected by the flow field $\mathbf{v}(\mathbf{x})=\frac{\mathbf{u}(\mathbf{x})}{\rho(\mathbf{x})}$. Particles were integrated using the same third-order Runga-Kutta scheme as for the fields. The field values at the particle positions $\mathbf{x}$ were evaluated by a linear interpolation, since the density has 


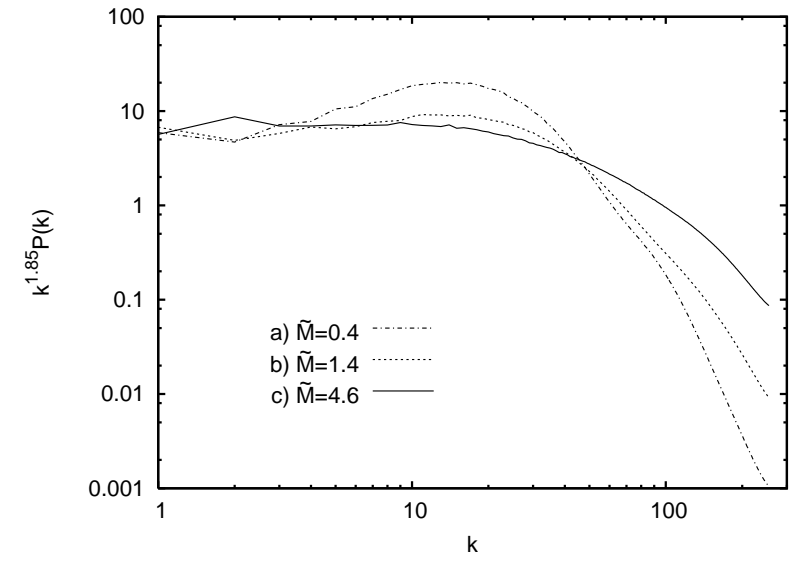

FIG. 1: Compensated velocity spectra

to be positive even in regions where strong shocks cause steep gradients close to flat areas. Higher order interpolators here tend to undershoot which leads to artificial particle reflection. After another dynamical time $t_{d y n}$ to assure that the particle statistics has reached a stationary state, data were analyzed both for Eulerian and the Lagrangian statistics.

Eulerian statistics: To characterize the flows we measured some specific values and show velocity-spectra and mass-density PDFs in the turbulent regime (see Figs. 1, 2 and Table I). In fully developed isotropic turbulence, the energy brought into the system at large scales should cascade through the scales and finally dissipate at small scales, known as the Richardson cascade of energy. In stationary turbulent flows, the energy injection equals the dissipation. Therefore, the mean energy dissipation $\varepsilon$ can be obtained from the mean energy input $\left\langle\partial_{t} E_{k}\right\rangle$ of the driving force $\mathbf{k}$ averaged over several dynamical times. Normalized to the mean kinetic energy in the box $\varepsilon / E_{\text {kin }}$ we see in Table \ that dissipation rises with the Mach number. In order to quantify the Eulerian compressibility the dimensionless ratio $\mathcal{C}=\left\langle\left(\partial_{i} v_{i}\right)^{2}\right\rangle /\left\langle\left(\partial_{i} v_{j}\right)^{2}\right\rangle$ is measured which is zero for solenoidal and one for potential flows. This quantity also shows a dependence of Mach number. Finally we give an estimate for the integral scale Reynolds number $R=l_{0} v_{0} / \nu$ defined by the integral length scale $l_{0}=2 / 3 E_{\text {kin }}^{3 / 2} / \varepsilon$. Here, $v_{0}$ is the root mean square velocity fluctuation, which in isothermal flows is the same as the mean Mach number, and the dynamical viscosity $\nu$ is given by $\nu=\eta^{4 / 3} \varepsilon^{1 / 3}$. The Kolmogorov dissipation scale $\eta$ was estimated by the discretization scale $\eta=\Delta x=L / \mathcal{N}$. Please note, that this is a conservative estimate since in spectral simulations of incompressible flows the dissipation scale $\eta$ is normally half the grid spacing. Only for high Mach number flows a Reynolds number can be reached which is comparable to the one obtained from spectral simulations of incompressible flows with the same resolution. The power spec-

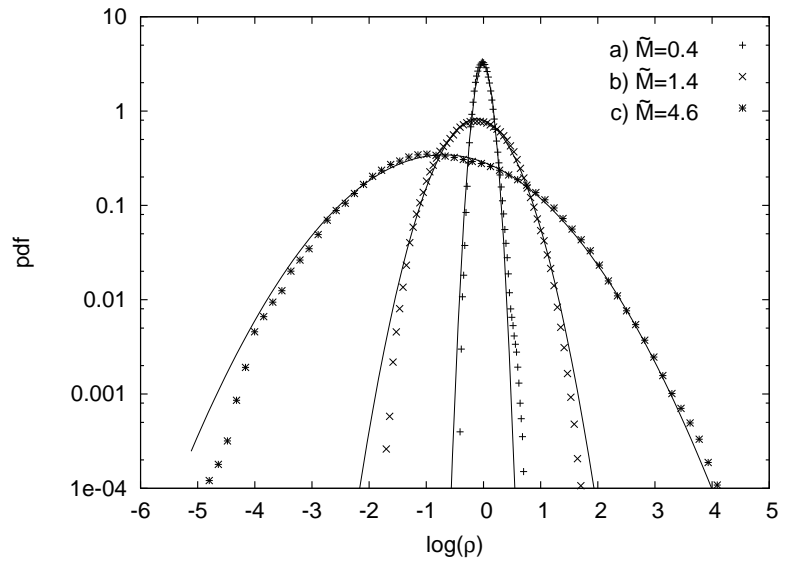

FIG. 2: PDF of logarithm of mass density. Solid lines are normal distribution with mean and variance obtained from data.

trum of the velocity fluctuations steepens with increasing Reynolds number reflecting the occurrence of strong shocks. The slope of the spectrum in the inertial range drops to a value, which is compatible to $P(k) \sim k^{-1.85}$ for run c. This is in agreement with the simulations of Kritsuk et al. [12] and Boldyrev et al. [13]. Although the latter is obtained from MHD simulations, it just reveals the dominance of strong shocks that converge to Burgerslike spectra $\sim k^{-2}$ for even higher Mach-numbers.

In the isothermal case the mass density PDF $R(\rho)$ should follow a log-normal distribution as discussed in [14, 15]. In Fig. 2 we compared the density PDF of the numerical simulations with normal distributions with mean and variance obtained from the numerical data which confirms the reasoning of [14, 15].

Distribution of tracer particles: To obtain the PDF of the spatial distribution of the tracer particles we divided the computational box into $n=40^{3}$ equally spaced subdomains and counted the particles in them. The number of boxes with $k$ particles gave the PDF of the tracers as a discrete distribution $T(k)$. Since the particles were initially randomly distributed, their PDF started from a Poissonian

$$
T_{\text {init }}(k)=P_{\lambda}(k)=\frac{\lambda^{k}}{k !} e^{-\lambda}
$$

with $\lambda=N / n$ and changed in time towards a stationary distribution.

TABLE I: Characteristic quantities for the three runs

\begin{tabular}{ccccc}
\hline \hline Run & Mach & $\mathcal{C}$ & $\varepsilon / E_{\text {kin }}$ & Reynolds number \\
\hline $\mathrm{a}$ & 0.4 & 0.07 & 0.31 & 250 \\
$\mathrm{~b}$ & 1.4 & 0.26 & 0.47 & 700 \\
$\mathrm{c}$ & 4.6 & 0.50 & 0.95 & 1756 \\
\hline \hline
\end{tabular}


To understand the relation between the mass density PDF and the particle distribution, we first consider an incompressible flow. The PDF of $\rho(\vec{x})=\rho_{0}=$ const is

$$
R_{\text {incomp }}(\rho)=\delta\left(\rho-\rho_{0}\right) .
$$

In this case the particle distribution stays the same for all times and the expectation values $\mathbb{E}\left(R_{\text {incomp }}\right)=\rho_{0}$ and $\mathbb{E}(T)=\mathbb{E}\left(T_{\text {init }}\right)=N / n$ are constant. The appropriate translation between the mass density PDF and the particle distribution is a convolution which maps the $\delta$-peak to the Poisson-distribution and matches the expected values:

$$
\begin{aligned}
\hat{T}(k) & =\left(R \circledast P_{\lambda}\right)(k) \\
: & =\int P_{\lambda=\frac{\mathbb{E}(T)}{\mathbb{E}(R)} \rho} \mathrm{d} \mu(\rho) \\
& =\int P_{\lambda=\frac{\mathbb{E}(T)}{\mathbb{R}(R)} \rho}(k) R(\rho) \mathrm{d} \rho .
\end{aligned}
$$

It is easy to see that this convolution works for the incompressible case. From that it is suggesting to take the same convolution for compressible turbulence with the measured $R(\rho)$ instead of the delta peak in the incompressible case. In this way the convolution generates a discrete particle distribution from the continuous distribution of density. In Fig. 3 we show the PDFs for run $\mathrm{a}$ and $\mathrm{c}$ (The PDFs for run b agree similarly well, but are omitted here). The solid lines $\hat{T}(k)$ are the result of the convolution of the measured $R(\rho)$ with the Poisson distribution. It reproduces in all cases nearly exactly the measured particle distribution $T(k)$.

In order to obtain an analytical expression, we use the relation between the deviation $\sigma$ of $\rho$ and the r.m.s. Mach number $M$ for isothermal supersonic turbulence introduced by Passot and Vazquez-Semadeni [14] and Nordlund and Padoan [15, 16]:

$$
\sigma^{2}=\beta^{2} M^{2} \text {. }
$$

This translates to the relation

$$
\sigma_{s}^{2}=\ln \left(1+\beta^{2} \frac{M^{2}}{\mathbb{E}^{2}(R)}\right)
$$

of the standard deviation $\sigma_{s}$ of $\ln \rho$ and the Mach number $M$.

If we convolve this lognormal distribution with a Poisson-distribution using eqn. (2) we obtain

$\hat{T}(k)=\frac{E^{k}}{\sqrt{2 \pi} \sigma_{s}} \frac{1}{k !} \int_{0}^{\infty} \rho^{k-1} \exp \left(-\frac{(\ln \rho-\mu)^{2}}{2 \sigma_{s}^{2}}-E \rho\right) \mathrm{d} \rho$

with

$$
E=\frac{\mathbb{E}(T)}{\mathbb{E}(R)}
$$

Hence, we can describe the particle-distribution with only one fit parameter $\beta$. In Fig. 4 we show the agreement between the particle-distribution and this theoretical PDF.
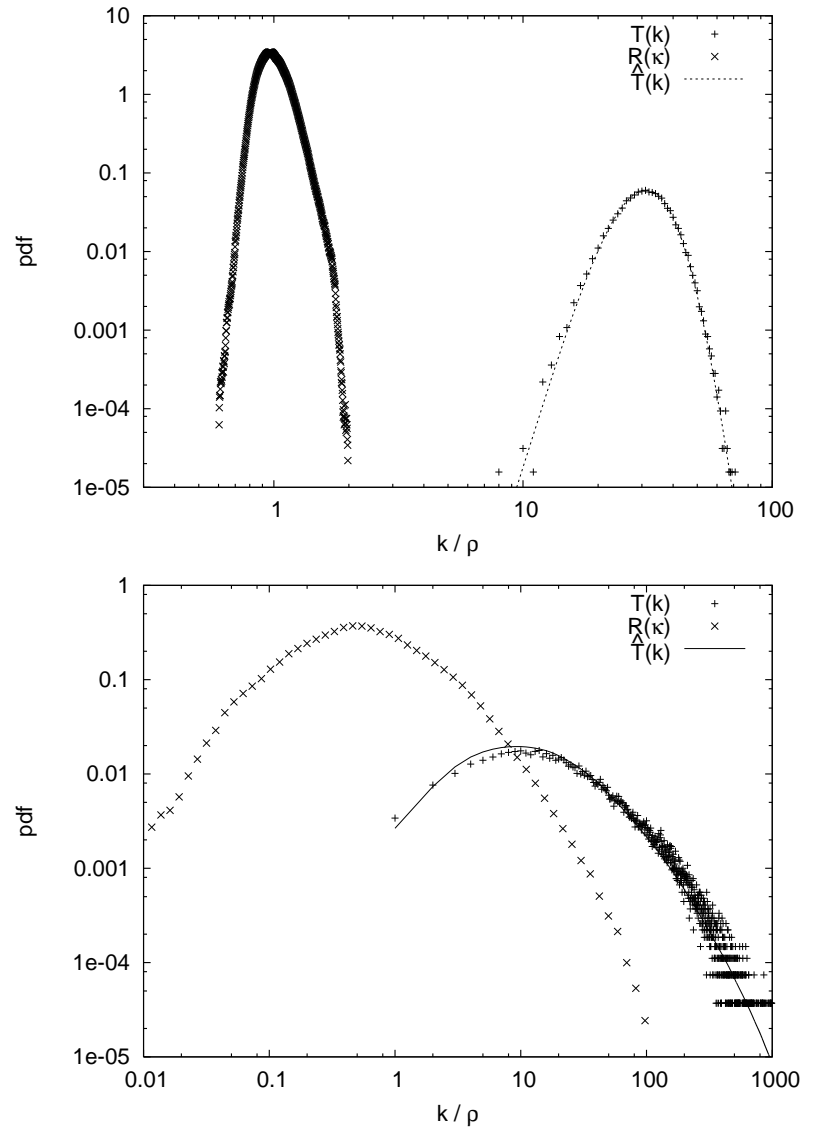

FIG. 3: The convolution operator maps the mass density PDF $R(\rho)$ on the particle distribution $\hat{T}(k)$ in good agreement with the measurement $T(k)$. Top: run a, bottom: run $\mathrm{c}$

This figure was obtained with a value of $\beta \simeq 0.37$. This value lies somewhere in between the value $\beta=0.26$ of the recent simulations of Kritsuk et al. 17 and $\beta=0.5$ of the MHD simulations of Padoan et al. [16].

Structuring time After this findings we can determine how long it takes for the particles to form global structures. This time $t_{S}$ is equivalent to the time in which the particle PDF goes from the initial Poissonian to the final distribution given in eqn. (44). To estimate this time we define an operator $\Lambda[T]$ on the particle distribution $T$ which assigns the initial Poisson distribution $T=P(k)$ to 1.0 and the final stationary distribution $T=\hat{T}(k)$ to 0 :

$\sum_{k}((1-\Lambda[T]) \cdot \hat{T}(k)+\Lambda[T] \cdot P(k)-T(k))^{2} \rightarrow \operatorname{Min}$

where the value $\Lambda[T]$ is obtained from the minimization procedure. A plot of the temporal development of $\Lambda[T]$ is shown is Fig. 5

To give an estimate for the time scale $t_{S}$ of the particle clustering, an exponentially damped oscillating function is fitted to the curve $\Lambda[T](t)$ with decay proportional to $\exp \left(-t / t_{S}\right)$. In Table II values for this structuring 


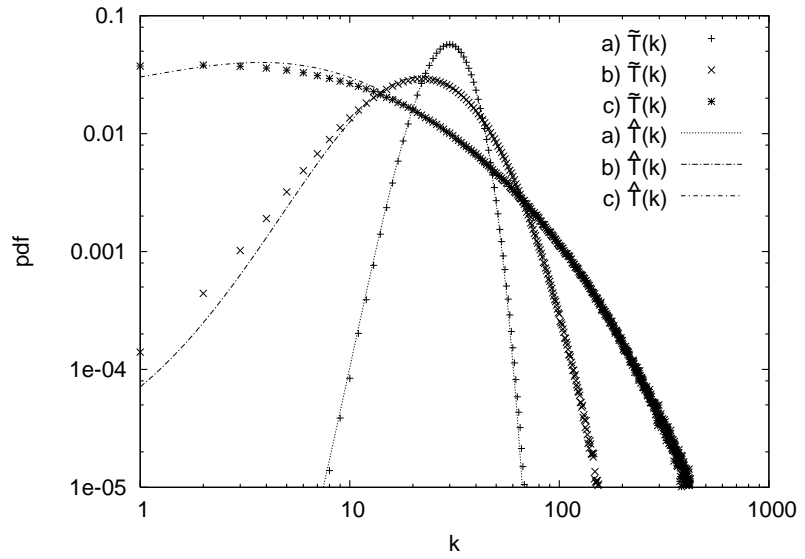

FIG. 4: Particle distribution averaged over several time steps $\tilde{T}(k)$ and the theoretical distribution $\hat{T}(k)$ obtained from equation (4) with $\beta=0.37$.

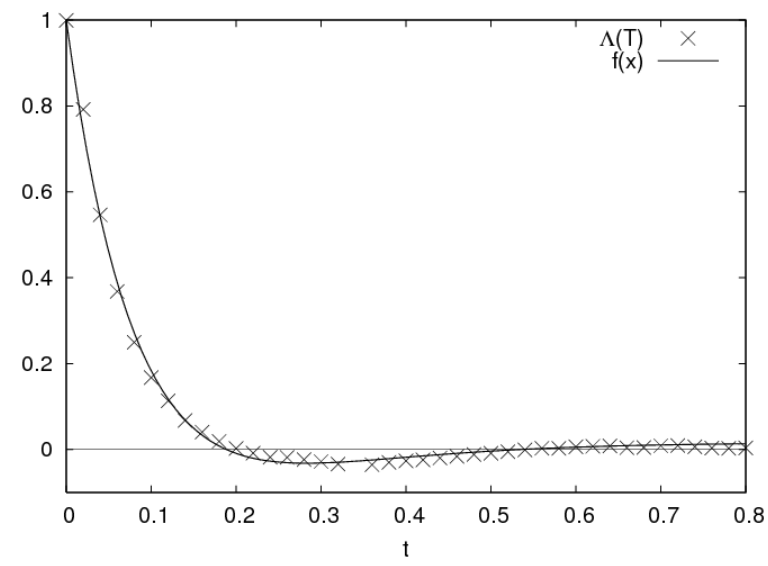

FIG. 5: Transition of the particle distribution from the Poissonian to the $\hat{T}(k)$-distribution characterized by $\Lambda[T](t)$ and compared to the exponential fit $f(t)$.

time $t_{S}$ as well as the dynamical time $t_{d y n}$ are given. The comparison shows that the structuring time is significantly shorter than the dynamical time, although the ratio $t_{S} / t_{d y n}$ grows slightly with increasing Mach number.

TABLE II: Comparison between the dynamical time $t_{d y n}$ and the structuring time $t_{S}$

\begin{tabular}{cccc}
\hline \hline Run & Mach & $t_{\text {dyn }}$ & $t_{S}$ \\
\hline $\mathrm{a}$ & 0.4 & 7.8 & 1.0 \\
$\mathrm{~b}$ & 1.4 & 2.2 & 0.33 \\
$\mathrm{c}$ & 4.6 & 0.62 & 0.11 \\
\hline \hline
\end{tabular}

Conclusions and outlook: In this Letter we have estab- lished a direct relationship between the particle distribution and the PDF of the density fluctuations in isothermal compressible turbulence. The analytical expression for the particle distribution confirms the relation between the standard deviation of $\ln \rho$ and the mean Mach number. The proportionality constant $\beta$ was obtained from the particle distribution. The Lagrangian viewpoint offers further studies on the formation of density structures. In this paper, we studied only the low order statistics. The next natural step is to consider higher order moments of the particle number in a given ball of radius $r$. A first step in this direction for an unrealistic compressible Kraichnan flow was done in Bec et al. [18].

Acknowledgments. - We'd like to acknowledge interesting discussions with R. Friedrich and J. Schumacher. Computations were performed on a Linux-Opteron cluster supported by HBFG-108-291. This work benefited from support through DFG-GK 1051 and DFG-SFB 591.

[1] P. Padoan and A. Nordlund, ApJ 576, 870 (2002).

[2] S. Ott and J. Mann, J. Fluid Mech. 422, 207 (2000).

[3] A. L. Porta, G. Voth, F. Moisy, and E. Bodenschatz, Phys. Fluids 12, 1485 (2000).

[4] G. Voth, A. L. Porta, A. Crawford, J. Alexander, and E. Bodenschatz, Rev. Sci. Instr. 12, 4348 (2001).

[5] A. L. Porta, G. V. A. Crawford, J. Alexander, and E. Bodenschatz, Nature 409, 1017 (2001).

[6] N. Mordant, P. Metz, O. Michel, and J.-F. Pinton, Phys. Rev. Lett. 87, 214501 (2001).

[7] G. B. L. Biferale, A. Celani, B. Devinish, A. Lanotte, and F. Toschi, Phys. Rev. Lett. 93, 064502 (2004).

[8] H. Homann, R. Grauer, A. Busse, and W. Müller, J. Plasma Phys, published online doi: 10.1017/S0022377807006575 (2007).

[9] J. Dreher and R. Grauer, Parallel Computing 31, 913 (2005).

[10] A. Kurganov and D. Levy, SIAM J. Sci. Comput. 22, 1461 (2000).

[11] C. Shu and S. Osher, J. Comput. Phys. 77, 439 (1988).

[12] A. G. Kritsuk, M. L. Norman, and P. Padoan, ApJ 638, 25 (2006).

[13] S. Boldyrev, A. Nordlund, and P. Padoan, ApJ 573, 678 (2002).

[14] T. Passot and E. Vásquez-Semadeni, Phys. Rev. E 58, 4501 (1998).

[15] Å. Nordlund and P. Padoan, in Interstellar Turbulence, Proceedings of the 2nd Guillermo Haro Conference (Cambridge University Press, 1999).

[16] P. Padoan, A. Nordlund, and B. Jones, MNRAS 288, 145 (1997).

[17] A. G. Kritsuk, M. L. Norman, P. Padoan, and R. Wagner, ApJ, preprint doi:10.1086/519443 (2007).

[18] J. Bec., K. Gawedzki, and P. Horvai, PRL 92, 224501 (2004). 